\title{
Statistical assessment of a laboratory method for growing biofilms
}

Correspondence

Martin A. Hamilton

marty_h@erc.montana.edu

Received 15 October 2004

Revised 6 December 2004

Accepted 10 December 2004

\author{
Darla M. Goeres, ${ }^{1}$ Linda R. Loetterle, ${ }^{1}$ Martin A. Hamilton, ${ }^{1}$ Ricardo Murga, ${ }^{2}$ \\ Douglas W. Kirby ${ }^{3}$ and Rodney M. Donlan ${ }^{2}$ \\ ${ }^{1}$ Center for Biofilm Engineering, Montana State University, Bozeman, MT 59717-3980, USA \\ ${ }^{2}$ Biofilm Laboratory, Division of Healthcare Quality Promotion, Centers for Disease Control and \\ Prevention, Atlanta, GA 30333, USA \\ ${ }^{3}$ Scientific Resources Program, Centers for Disease Control and Prevention, Atlanta, \\ GA 30333, USA
}

Microbial biofilms have been grown in laboratories using a variety of different approaches. A laboratory biofilm reactor system, called the CDC biofilm reactor (CBR) system, has been devised for growing biofilms under moderate to high fluid shear stress. The reactor incorporates 24 removable biofilm growth surfaces (coupons) for sampling and analysing the biofilm.

Following preliminary experiments to verify the utility of the CBR system for growing biofilms of several clinically relevant organisms, a standard operating procedure for growing a Pseudomonas aeruginosa biofilm was created. This paper presents the results of a rigorous, intra-laboratory, statistical evaluation of the repeatability and ruggedness of that procedure as well as the results of the experiments with clinically relevant organisms. For the statistical evaluations, the outcome of interest was the density (c.f.u. $\mathrm{cm}^{-2}$ ) of viable $P$. aeruginosa. Replicate experiments were conducted to assess the repeatability of the log density outcome. The mean $P$. aeruginosa $\log _{10}$ density was $7 \cdot 1$, independent of the coupon position within the reactor. The repeatability standard deviation of the log density based on one coupon per experiment was 0.59 . Analysis of variance showed that the variability of the log density was $53 \%$ attributable to within-experiment sources and $47 \%$ attributable to between-experiments sources. The ruggedness evaluation applied response-surface design and regression analysis techniques, similar to those often used for sensitivity analyses in other fields of science and engineering. This approach provided a quantitative description of ruggedness; specifically, the amount the log density was altered by small adjustments to four key operational factors - time allowed for initial surface colonization, temperature, nutrient concentration, and fluid shear stress on the biofilm. The small size of the regression coefficient associated with each operational factor showed that the method was rugged; that is, relatively insensitive to minor perturbations of the four factors. These results demonstrate that the CBR system is a reliable experimental tool for growing a standard biofilm in the laboratory and that it can be adapted to study several different micro-organisms.

\section{INTRODUCTION}

Many in vitro systems have been developed for growing and testing microbial biofilms. These include simple batch/ static systems (O’Toole \& Kolter, 1998), batch systems with introduced shear (Ceri et al., 1999), flow cells (Mittelman et al., 1992), perfused biofilm fermenters (Allison et al., 1999), and systems that can be operated under continuousflow conditions such as the rotating-disc reactor (Zelver et al., 1999), the modified Robbins device (Nickel et al.,

Abbreviations: CBR, CDC biofilm reactor; SOP, standard operating procedure.
1985; Kharazmi et al., 1999) and the annular reactor (Camper et al., 1996). These systems may operate under batch or continuous-flow configurations, and generally provide a surface that can be removed and examined once it is colonized to assess biofilm formation. Donlan et al. (2002) developed a reactor (CDC biofilm reactor, CBR) that incorporated 24 removable biofilm growth surfaces allowing biofilm formation under moderate to high shear in batch or continuous-flow conditions. Studies that utilized this reactor showed that it could be used for detecting biofilm formation, characterizing biofilm structure (Donlan et al., 2004) and assessing the effect of antimicrobial agents on the biofilm (Donlan et al., 2002). However, a thorough 
statistical evaluation that incorporated an assessment of the reproducibility, repeatability and ruggedness of this system had not been conducted.

In this study, preliminary experiments were performed to generate biofilms of three clinically relevant microorganisms, Pseudomonas aeruginosa, Klebsiella pneumoniae and Streptococcus pneumoniae. Then a standard operating procedure (SOP) was created for growing a $P$. aeruginosa biofilm. This procedure was incorporated into an intralaboratory evaluation to determine the repeatability and ruggedness of the method. We present a description of the CBR system, preliminary results on biofilms grown in that system, an SOP for P. aeruginosa and the results of a study designed specifically to evaluate the repeatability and ruggedness of the SOP.

\section{METHODS}

Description of the CDC biofilm reactor (CBR). The CBR (Biosurface Technologies, Bozeman, MT, USA) consisted of a one-litre glass vessel with an effluent spout positioned to provide approximately $350 \mathrm{ml}$ operational fluid capacity (Fig. 1). An ultrahigh-molecular-mass polyethylene top supported eight independent and removable polypropylene rods, a medium-inlet port, and a gasexchange port. Each rod held three removable coupons (biofilm growth surfaces) for a total of 24 sampling opportunities. Each coupon was a disk $(1.27 \mathrm{~cm}$ diameter, $0.3 \mathrm{~cm}$ thick). The glass vessel was placed on a digitally controlled stir plate to provide constant rotation of the baffled stir bar at a designated speed (r.p.m.). Rotation of the baffle provided constant mixing and consistent shear

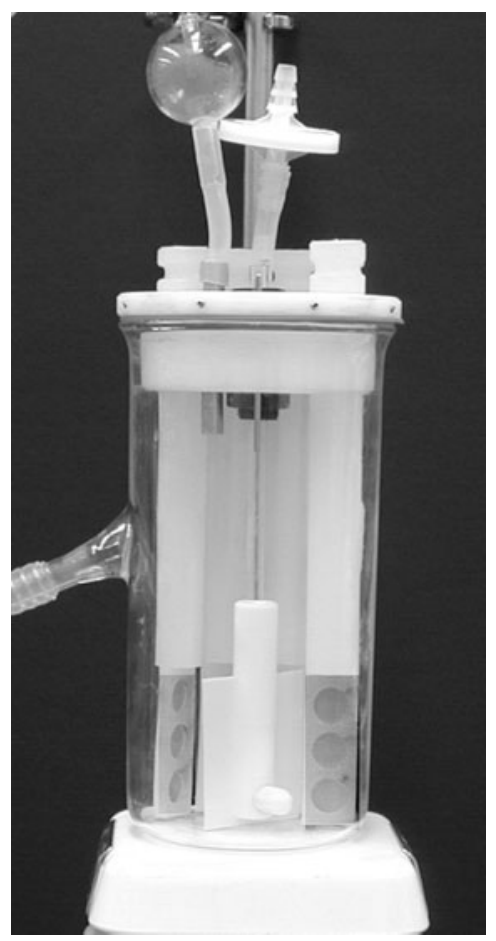

Fig. 1. The CDC biofilm reactor (CBR). Rods have been removed to expose the baffled stirrer. to the coupon surface. The intensity of shear experienced by the coupons was a function of the speed at which the baffle rotated and the distance from the outer edge of the baffle to the coupon face. Sterile medium was pumped into the glass vessel using a peristaltic pump (Cole Parmer, Vernon Hills, IL, USA). The CBR was operated as a continuous-flow stirred-tank reactor, i.e. nutrients continuously flowed into and out of the reactor at a chosen, fixed rate (Characklis \& Marshall, 1990). For experiments in which an increased reactor temperature was required, the glass vessel was placed into a water bath. Temperature was maintained by a digitally controlled temperature controller (Digi-sense, model no. 89000-00; Cole Parmer) and heating element (Heet-O-Matic, model 324, Cole Parmer).

Preliminary experiments. $P$. aeruginosa (ATCC 7700) and $K$. pneumoniae (CDC culture no. DMDS Lab 92-08-28a) were grown separately on plates of R2A agar (Difco) passaged twice from frozen stocks, incubated at $30^{\circ} \mathrm{C}$ for $24 \mathrm{~h}$, then suspended in phosphatebuffered saline (PBS) to a concentration equivalent to a 0.5 McFarland standard. Stainless steel $316 \mathrm{~L}$ coupons were cleaned in $70 \%$ ethanol, rinsed in filter-sterilized reverse osmosis (RO) water, placed in the CBR, and then the assembled reactor was sterilized by autoclaving. The sterile reactor was filled with filter-sterilized medium containing $0.05 \mathrm{~g}$ yeast extract, proteose peptone no. 3, Casamino acids, glucose, $0.03 \mathrm{~g}$ sodium pyruvate, $0.03 \mathrm{~g}$ dibasic potassium phosphate, and $0.005 \mathrm{~g}$ magnesium sulfate (all from Difco) per litre of RO water. After inoculation, the reactor was placed in a water bath to maintain a temperature of $30^{\circ} \mathrm{C}$. The water bath was placed onto the surface of a mixing plate set to provide constant mixing at 100 r.p.m. The system was operated under batch conditions for $72 \mathrm{~h}$ then under continuous-flow conditions by pumping a 1/10 dilution of the medium defined above at a flow rate of $1 \mathrm{ml} \mathrm{min}^{-1}$ (providing a residence time of $6.6 \mathrm{~h}$ ) for $24 \mathrm{~h}$ prior to sampling all 24 coupons. The batch conditions provided additional time for the attachment of organisms prior to initiation of flow. The biofilm growth protocol was based upon Murga et al. (2001).

S. pneumoniae (clinical isolate from the Boston's Children's Hospital, Boston, MA, USA, provided by Paul Edmonds, Georgia Institute of Technology, Atlanta, GA, USA) was transferred from a frozen stock onto Trypticase soy agar containing $5 \%$ sheep's blood (blood agar) (BD Microbiology Systems) and incubated at $35^{\circ} \mathrm{C}$ in a $5 \% \mathrm{CO}_{2}$ incubator overnight. A single colony was picked and inoculated into a $10 \mathrm{ml}$ tube of brain heart infusion (BHI) broth (Difco), and incubated for $12 \mathrm{~h}$ at $35^{\circ} \mathrm{C}$ in a $5 \% \mathrm{CO}_{2}$ incubator. For the $S$. pneumoniae experiments, the CBR contained $1.27 \mathrm{~cm}$ diameter Teflon coupons. The reactor was sterilized in an autoclave, then filled with $400 \mathrm{ml}$ of filter-sterilized full strength BHI broth. Nine millilitres of the $12 \mathrm{~h}$ culture was added to the reactor, providing $2 \cdot 13 \times 10^{7}$ c.f.u. $\mathrm{ml}^{-1}$ in the reactor as determined by plating on blood agar incubated at $35^{\circ} \mathrm{C}$ in a $\mathrm{CO}_{2}$ incubator. A filter-sterilized mixture of $85 \% \mathrm{~N}_{2}, 10 \%$ $\mathrm{CO}_{2}$ and $5 \% \mathrm{O}_{2}$ was continually supplied to the CBR to provide an atmosphere of supplemental $\mathrm{CO}_{2}$. The entire reactor was placed into a heated water bath to maintain a temperature of approximately $35^{\circ} \mathrm{C}$ for the duration of the experiment. The system was operated in batch mode for $12 \mathrm{~h}$, then in continuous-flow by pumping a $1 / 10$ dilution of BHI broth at a flow rate of $0.5 \mathrm{ml} \mathrm{min}$ m $^{-1}$ (providing a residence time of $13 \cdot 3 \mathrm{~h}$ ) for $24 \mathrm{~h}$ prior to sampling coupons.

In each preliminary experiment, all 24 coupons in the reactor were sampled and analysed. Each rod was placed into a biological safety cabinet and three coupons from one rod were aseptically removed. Each coupon was rinsed twice in Butterfield buffer, $\mathrm{pH} 7 \cdot 2$ (cat. no. 298267, containing $0 \cdot 4 \mathrm{~g}$ monobasic potassium phosphate $1^{-1}, 0 \cdot 1 \%$ peptone, $2.0 \%$ polysorbate 80 ; Becton Dickinson) to remove planktonic cells and placed into a tube containing $10 \mathrm{ml}$ of PBS. Biofilm bacteria were recovered by subjecting coupons to three 
alternating $30 \mathrm{~s}$ cycles of sonication at a frequency of $42 \mathrm{kHz}$ (model 2510 sonicating water bath; Branson), followed by vortexing (Vortex Genie 2; Scientific Products). The removed biofilm was disaggregated by homogenizing the suspension with a tissue homogenizer (Polyscience Tissue Homogenizer model K-120; Polysciences) at 16000 r.p.m. for $60 \mathrm{~s}$. The disaggregated biofilm was then processed to quantify the number of viable cells. For $P$. aeruginosa and $K$. pneumoniae, this entailed spread-plating serial dilutions of the suspension onto R2A agar (Difco), incubating the plates at $35^{\circ} \mathrm{C}$ for $48 \mathrm{~h}$ and counting the colonies. For $S$. pneumoniae, the diluted suspension was spreadplated onto blood agar and colonies were counted after incubating for $24 \mathrm{~h}$ at $35^{\circ} \mathrm{C}$ in a $\mathrm{CO}_{2}$ incubator.

Following the preliminary experiments, the experimental protocol was modified to provide conditions optimal for the $P$. aeruginosa strain used in the ruggedness tests. The sample and analysis steps were also evaluated and modified (see the standard operating procedure below).

Standard operating procedure (SOP). Polycarbonate coupons were sonicated for $30 \mathrm{~s}$ in a detergent solution (a 1-2\% solution of Micro-90; International Products Corporation). Then each was rinsed and sonicated for another $30 \mathrm{~s}$ in reagent-grade water. Alternate rinsing and sonication was repeated until no soap was left on the coupon surface. The coupons were then soaked for $2 \mathrm{~h}$ in $2 \mathrm{M} \mathrm{HCl}$, rinsed, and allowed to air dry prior to use. One coupon was positioned into each hole of the reactor rods so that the face of the coupon was flush with the rod surface that faced the baffle. A set screw was tightened to hold the coupon in place. The reactor system was then assembled and $500 \mathrm{ml}$ of $300 \mathrm{mg}$ tryptic soy broth (TSB) $1^{-1}$ (Difco) was added. The assembled system, minus the pump and stir plate, was autoclaved.

After the system had cooled to $23^{\circ} \mathrm{C}$, the glass vessel was set on a digital stir plate and inoculated with $1 \mathrm{ml}$ of a $10^{8}$ c.f.u. $\mathrm{ml}^{-1}$ suspension of $P$. aeruginosa (ATCC 700888). The $P$. aeruginosa suspension was prepared by inoculating $100 \mathrm{ml}$ of broth $\left(300 \mathrm{mg}\right.$ TSB $\mathrm{l}^{-1}$ ) with a single colony collected from a bacterial isolation plate. The suspension was incubated for $18-24 \mathrm{~h}$ at $37^{\circ} \mathrm{C}$ in a shaker. Immediately after the addition of $P$. aeruginosa to the reactor, the stir plate was set to rotate at 125 r.p.m. The biofilm was allowed to establish for $24 \mathrm{~h}$ in batch phase while the baffle rotated. A $24 \mathrm{~h}$ continuous-flow phase followed immediately. While the baffle continued to rotate, a $100 \mathrm{mg}$ $\mathrm{TSB} 1^{-1}$ nutrient broth was pumped into the reactor at a rate equal to $11 \cdot 7 \mathrm{ml} \mathrm{m^{-1 }}$, resulting a $30 \mathrm{~min}$ residence time.

The stir plate and pump were turned off after $24 \mathrm{~h}$ of continuous flow and the biofilm was harvested from a predetermined number of randomly selected coupons. For each selected coupon, the rod holding that coupon was removed through the top of the reactor. The set screw that held the coupon in place was loosened and the coupon was removed using a flame-sterilized haemostat. Care was taken not to disturb the surface of the coupon facing the baffle because that surface held the biofilm sample that was analysed. Following the procedures described by Zelver et al. (1999), the biofilm was then scraped from the surface, homogenized to create a uniform cell suspension, serially diluted, plated on R2A agar, incubated for $18-24$ h at $37 \pm 2{ }^{\circ} \mathrm{C}$ and viable cell numbers (c.f.u.) were counted. In brief, to remove the biofilm from the coupon, the coupon was held with a sterile clamp. Using a sterile applicator stick, the surface of the coupon was thoroughly scraped for about $1 \mathrm{~min}$. During that time, the stick was occasionally stirred in $9 \mathrm{ml}$ sterile buffered water to remove attached material. After sufficient scraping, the coupon surface was rinsed with $1 \mathrm{ml}$ sterile buffered water. The final volume in the sample test tube was $10 \mathrm{ml}$. Then a sterile homogenizer probe was inserted into the homogenizer and the sample tube was homogenized at $\sim 20500$ r.p.m. for $60 \mathrm{~s}$. The disaggregated biofilm was then processed to quantify the number of viable cells. This entailed drop-plating serial dilutions of the suspension onto R2A agar (Difco), incubating the plates at $37^{\circ} \mathrm{C}$ for $24 \mathrm{~h}$, and counting the colonies (Herigstad et al., 2001).

The key measurement was the $\log _{10}$ density, where density was expressed in units of c.f.u. $\mathrm{cm}^{-2}$, for each sampled coupon, calculated with the following equation:

$\log$ density $=\log _{10}($ mean c.f.u. per plate $)+\log _{10}($ vol. scraped into $)+$ $\log _{10}$ (dilution) $-\log _{10}$ (vol. plated $)-\log _{10}$ (area of coupon face $)$

The 'area of coupon face' was $1 \cdot 267 \mathrm{~cm}^{2}$. For this and all subsequent statistical calculations performed on the $\log _{10}$ scale, five or more significant figures past the decimal point were carried; rounding occurred only at the conclusion of the calculations.

Ruggedness and repeatability evaluation study. A series of experiments were conducted to estimate the repeatability standard deviation, denoted by $s_{\mathrm{r}}$, and the regression coefficients that measure the ruggedness of the CBR SOP. In these experiments, the SOP was not followed exactly; instead, some of the operational factors were purposely varied slightly. For example, some experiments were conducted at $20^{\circ} \mathrm{C}$ rather than at the SOP temperature of $23^{\circ} \mathrm{C}$. For the ruggedness tests, we purposely altered the settings of four operational factors - temperature, r.p.m. of the rotating baffle, time in batch, and nutrient concentration during continuous flow. Three settings were selected for each factor (Table 1).

A complete factorial experimental design for testing all combinations of the settings would entail $3^{4}=81$ experiments plus some replicates for purposes of calculating $s_{\mathrm{r}}$. Instead of running a complete factorial design, a response-surface design was used. Experimentation was conducted in two phases, with replicates in each phase for purposes of calculating $s_{\mathrm{r}}$. The first phase used a fractional factorial design (half fraction of a $2^{4}$ factorial) plus replicate runs at the SOP (AOAC, 1998 - Appendix C). The data from the first phase were evaluated to see if the log density was unaffected by any of the factors; if so, those factors could be dropped from the next phase. All of the factors had an effect; therefore none were dropped. The second phase consisted of one-at-a-time experiments, where three of the factors were held at their SOP settings and the fourth factor was at a non-SOP setting. Several experiments in this phase were conducted in which only SOP settings were used. For final analysis, data from both phases were combined and the results were based on a total of 21 separate experiments with five or six randomly sampled coupons in each experiment, resulting in a total of 124 sampled coupons.

Ruggedness was quantified by the regression coefficients in a

Table 1. The three settings for each of the four operating conditions that were studied in the ruggedness test

\begin{tabular}{|lrrr|}
\hline Operating conditions & \multicolumn{3}{c|}{ Settings $^{*}$} \\
\cline { 2 - 4 } & Low & Medium & High \\
\hline Temperature $\left({ }^{\circ} \mathrm{C}\right)$ & 20 & $\mathbf{2 3}$ & 26 \\
Baffled stir bar rotation speed (r.p.m.) & $\mathbf{1 2 5}$ & 180 & 225 \\
Time in batch (h) & 4 & 18 & $\mathbf{2 4}$ \\
Nutrient $\dagger$ concn during continuous & 50 & $\mathbf{1 0 0}$ & 200 \\
flow $\left(\mathrm{mg} \mathrm{l}^{-1}\right.$ ) & & & \\
\hline
\end{tabular}

*Standard operating procedure (SOP) values are shown in bold.

$\dagger$ Tryptic soy broth. 
least-squares multiple regression analysis where the response variable was $\log$ density and the predictor variables were temperature, r.p.m., $\log _{10}$-transformed time in batch, and $\log _{10}$-transformed nutrient concentration during continuous flow. To do the analysis, the four predictor variables were entered as covariates into the General Linear model component of the analysis of variance (ANOVA) module in the computer software package Minitab (Release 13; Minitab, State College, PA, USA).

The ANOVA also provided a variance component analysis to assess the variance within experiments and the variance between experiments. The square root of the sum of those two variances was $s_{\mathrm{r}}$, which was interpreted as the typical difference, sign neglected, between the log density for a single (randomly chosen) experiment and the mean log density across many independent, identical (same operational factor settings) experiments.

\section{RESULTS}

\section{Preliminary experiments}

Preliminary testing demonstrated that the CBR, when operated with shear under a combination of batch and continuous-flow conditions, was capable of generating biofilms of three different organisms on replicate surfaces. Based on a sample of 24 coupons, the mean $\log _{10}$ density $( \pm \mathrm{SD})$ was $6 \cdot 77( \pm 0 \cdot 30) \log _{10}$ c.f.u. $\mathrm{cm}^{-2}$ for K. pneumoniae, $5 \cdot 33( \pm 0 \cdot 22) \log _{10}$ c.f.u. $\mathrm{cm}^{-2}$ for $S$. pneumoniae, and $5 \cdot 63( \pm 0 \cdot 17) \log _{10}$ c.f.u. $\mathrm{cm}^{-2}$ for P. aeruginosa.

\section{Coupon position}

Each rod in the CBR held three coupons in vertical alignment. Data from the preliminary experiments with $K$. pneumoniae, S. pneumoniae and $P$. aeruginosa were submitted to an analysis of variance. Neither the eight rods nor the three coupon positions significantly affected the mean log density $(P>0.50$ for each of the three organisms). For the SOP ruggedness test experiments, the mean differences in $\log$ densities were 0.18 (bottom minus middle), $0 \cdot 10$ (bottom minus top) and -0.08 (middle minus top). The mean log densities for the three positions were not significantly different $(P=0 \cdot 22)$. These results indicate that the 24 coupons in the reactor were equally representative of the log density.

\section{Repeatability}

For the SOP ruggedness test experiments, replicate experiments were conducted to allow assessment of repeatability of the SOP. The mean $\log _{10} P$. aeruginosa density (c.f.u. $\mathrm{cm}^{-2}$ ) was $7 \cdot 0590$, independent of coupon position within the reactor. The estimated within-experiment variance was $0 \cdot 1884$ (estimated with 103 degrees of freedom) and the estimated between-experiment variance was $0 \cdot 1656$ (estimated with 8 degrees of freedom). Therefore, $s_{\mathrm{r}}=(0 \cdot 1884+0 \cdot 1656)^{1 / 2}=0 \cdot 59$, of which $53 \%$ was attributable to within-experiment variation and $47 \%$ to between-experiment variation. This $s_{\mathrm{r}}$ pertained to a protocol that sampled only one coupon per experiment. The repeatability standard deviation for a protocol that requires sampling $\mathrm{n}$ coupons per experiment is

$S_{\mathrm{r}}=[(0 \cdot 1884 / n)+0 \cdot 1656]^{1 / 2}$

According to this equation, the $s_{\mathrm{r}}$ for the biofilm mean log density based on three coupons $(n=3)$ would equal $0 \cdot 48$, of which $27 \%$ is attributable to within-experiment variation and $73 \%$ to between-experiment variation. For the most intense sampling protocol possible, where all $n=24$ coupons in the reactor are sampled, $s_{\mathrm{r}}=0 \cdot 42$, which is $95 \%$ attributable to between-experiment variation.

Because there is an important between-experiment variance, it would be reasonable to choose an operating procedure that requires $m$ independent experiments, $n$ coupons per experiment. In this case, the log density estimate would be the mean $\log$ density across all $n \cdot m$ coupons, where the repeatability standard deviation is

$s_{\mathrm{r}}=[(0 \cdot 1884 /(n \cdot m))+0 \cdot 1656 / \mathrm{m}]^{1 / 2}$

\section{Ruggedness}

Equation (1) is the least-squares regression model for log density. It shows the estimated log density associated with any pattern of operating conditions, as long as those operating conditions are 'near' the SOP specifications. Fig. 2 shows the relationship between the predicted and observed $\log$ density for each coupon, where the predictions are based on equation (1). The correlation coefficient between the predicted and observed values (the multiple correlation coefficient) is $0 \cdot 89$, indicating that the regression model is a good fit to the log densities.

$$
\begin{aligned}
\log _{10}\left(\text { c.f.u. } \mathrm{cm}^{-2}\right)= & 7 \cdot 0590+0 \cdot 0191(\text { temp. }-23)- \\
& 0 \cdot 00867(\text { r.p.m. }-125)+ \\
& 1 \cdot 2014 \log _{10}(\text { time in batch } / 24)+ \\
& 2 \cdot 1258 \log _{10}(\text { nutrient concn } / 100)
\end{aligned}
$$

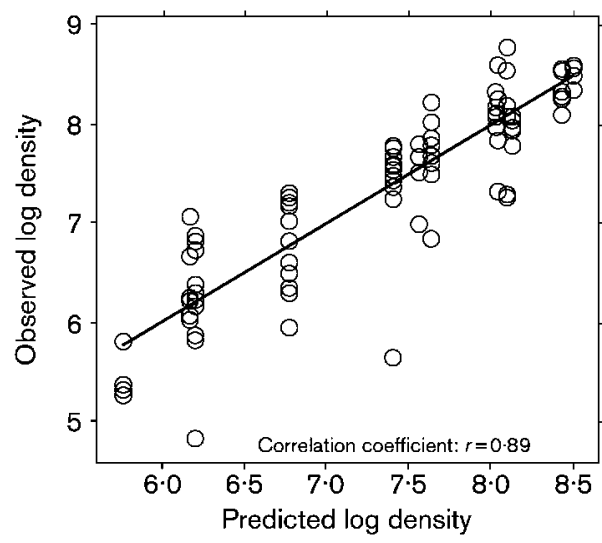

Fig. 2. Relationship between the estimated (equation 1) and observed biofilm log densities. The line of equality is shown. 
Equation (1) provides a quantitative ruggedness assessment because the sizes of the regression coefficients indicate the extent to which the associated condition affects the mean $\log$ density. If the experiment was conducted at a temperature $2{ }^{\circ} \mathrm{C}$ higher than specified in the SOP, the mean $\log$ density would be increased by only $0 \cdot 0191(2)=0 \cdot 04$. If the experiment was conducted with the baffle rotating at 10 r.p.m. faster than specified in the SOP, the mean $\log$ density would be decreased by only $0 \cdot 00867(10)=0 \cdot 09$. If the experiment was conducted with the time in the batch mode increased by $10 \%$ to $26 \cdot 4 \mathrm{~h}$ the mean $\log$ density would be increased by only $1 \cdot 2014\left[\log _{10}(1 \cdot 1)\right]=0 \cdot 05$. Finally, if the experiment was conducted with the nutrient concentration during the continuous flow mode decreased by $10 \%$ to $90 \mathrm{mg} \mathrm{TSB} \mathrm{ml}^{-1}$, the mean log density would be decreased by only $0 \cdot 10$ because $2 \cdot 1258\left[\log _{10}(0 \cdot 9)\right]=-0 \cdot 10$.

\section{DISCUSSION}

\section{Statistical evaluation}

Guidelines for evaluating a microbiological laboratory method usually include a list of desirable characteristics such as repeatability, reproducibility and ruggedness (Feldsine et al., 2002; ISO, 1993). A method is considered repeatable if independent repeats of the same experiment in the same laboratory produce nearly the same results. The conventional measure of repeatability is the standard deviation or some multiple of the standard deviation (ASTM, 2002a; Feldsine et al., 2002).

A method is reproducible if the same result occurs when the same experiment is run independently by different researchers in different laboratories. A collaborative study involving several laboratories is required to assess reproducibility. The results of the collaborative study are summarized conventionally by a standard deviation, called the reproducibility standard deviation, which can be no smaller, and is usually significantly larger, than the repeatability standard deviation (AOAC, 1995; ASTM, 2002a; Feldsine et al., 2002).

A rugged method is one for which the outcome is insensitive to minor perturbations of critical factors or conditions. There is no conventional quantitative measure for ruggedness, though several have been suggested (Thompson et al., 2002; AOAC, 1998 - Appendix C; ASTM, 2002b). One reason for conducting a ruggedness test is to provide a single laboratory approximation to a collaborative study. If the operational factors are changed by the amounts that one expects them to vary among laboratories, the standard deviation of the results should be similar to the reproducibility standard deviation. That expectation may be too optimistic. Although there could be considerable savings of resources if in fact a single laboratory ruggedness test could be substituted for a collaborative study, it is not possible at present to design such a test, due to lack of knowledge about all relevant factors (Thompson, 2000).
A ruggedness test can highlight the critical components of a laboratory method so that practitioners know which steps or conditions require special attention or which parameters should be optimized before a collaborative study is performed. Our informal examination of the literature indicates that ruggedness evaluations of new microbiological methods are seldom conducted. Moreover, we are unaware of other studies describing a quantitative assessment of the influence of individual operational factors on microbiological methods in general and biofilm methods in particular. The work presented here demonstrates that quantitative ruggedness evaluations of microbiological methods can be feasible and informative. The statistical design and analysis strategy presented here could also be used to evaluate other microbiological methods.

This study used a response-surface experimental design (ASTM, 2002b; NIST/SEMATECH, 2003), starting with a fractional factorial design (Youden \& Steiner, 1975), to identify the smallest number of experiments necessary for a multiple regression analysis. This two-step approach was efficient and provided the desired measure of the influence of each factor.

The choice of which operational factors to study and the range of settings for each factor were subjective steps in the ruggedness evaluation. It was unfeasible to study all operational factors; therefore, we relied on experience gained during the development of the reactor and SOP to choose the important factors for evaluation. The relevant settings for each factor were those that fell within the range expected when competent researchers faithfully followed the SOP. We observed responses at three or more levels of each factor. This made it possible to inspect the data for a potential nonlinear association between log density and the factor. There was no evidence of nonlinearity within the range of settings used for any of the four factors. The most reliable regression coefficient estimates occur when one chooses lower and upper settings of the operational factor as far apart as possible within the range of linearity (Thompson et al., 2002).

The interpretation of the influence of operational factors is complicated if there is an interaction, i.e. if the regression coefficient for one factor is dependent on one of the other factors. We did not expect interactive effects to occur within the ranges of factor settings investigated. However, it was unfeasible to run the large number of experiments required to check whether all potential interactions were in fact negligible. Note that the model of equation (1), which does not contain interaction terms, fits the data (Fig. 2).

\section{The CDC system}

Laboratory-grown biofilms are engineered to emulate a specific real-world environment. By altering parameters, such as flow dynamics and reactor configuration, it is possible to grow biofilms differing in structure (e.g. thickness) and function (e.g. nutrient consumption). A case in 
point is the effect of shear conditions on biofilm structure. Stoodley et al. (1999) showed that under high shear, biofilms consisted of dense elongated cell clusters while under low shear, biofilms were composed of less dense round cell clusters. Neither of these is the uniformly correct laboratory biofilm; each emulates a different growth environment. Because the choice of reactor affects the laboratory biofilm, it is important for the investigator to choose the appropriate reactor and growth conditions. The biofilm reactor (CBR) described in this paper provided a useful tool for growing repeatable biofilms under constant shear using a variety of organisms. By standardizing the design of the reactor, establishing an SOP, and performing multiple experiments, it was possible to conduct a statistical evaluation.

The ruggedness results showed that the baffle rotation speed could be an influential factor if it is not carefully controlled. For example, if the protocol was followed except that the stir plate was set so that the baffle rotated at a speed of 180 r.p.m. instead of 125 r.p.m. as specified by the protocol, the mean log density for a coupon would be estimated to be $6 \cdot 58$, a decrease of $0 \cdot 47$. We recommend that a digital stir plate be used with the CBR to provide that control.

The CBR can be operated under a wide range of controllable conditions. With modifications to the SOP, we believe it can be used to grow a standard biofilm for addressing diverse research questions.

\section{ACKNOWLEDGEMENTS}

This research was supported by the Montana Board of Research and Commercialization Technologies, the US Environmental Protection Agency (EPA), and the Industrial Associates of the Center for Biofilm Engineering. The views expressed here do not necessarily reflect the views of the US EPA. Use of trade names and commercial sources is for identification only and does not imply endorsement by the Public Health Service or the US Department of Health and Human Services. The laboratory experiments were ably conducted by Wes Bauman, Marion Clark and Andrew Anacker. Bryan Warwood, BioSurface Technologies, provided guidance in refining the reactor design so that the reactor would be both reliable and feasible to manufacture. Kelli Buckingham-Meyer, Joanna Heersink and Shamus McCarthy, all at the Center for Biofilm Engineering, provided technical assistance.

\section{REFERENCES}

Allison, D., Maira-Litran, T. \& Gilbert, P. (1999). Perfused biofilm fermenters. Methods Enzymol 310, 232-248.

AOAC (1995). Guidelines for collaborative study procedures to validate characteristics of a method of analysis. J AOAC Int 78, 143A-160A.

AOAC (1998). AOAC Peer-verified Methods Program Manual on Policies and Procedures. Gaithersburg, MD: AOAC International.

ASTM (2002a). E456-02: Standard terminology relating to quality and statistics. In Annual Book of ASTM Standards, vol. 14.02. West Conshohocken, PA: ASTM International.
ASTM (2002b). E1169-02: Standard guide for conducting ruggedness tests. In Annual Book of ASTM Standards, vol. 14.02. West Conshohocken, PA: ASTM International.

Camper, A., Jones, W. L. \& Hayes, J. T. (1996). Effect of growth conditions and substratum composition on the persistence of coliforms in mixed-population biofilms. Appl Environ Microbiol 62, 4014-4018.

Ceri, H., Olson, M. E., Stremick, C., Read, R. R., Morck, D. \& Buret, A. (1999). The Calgary Biofilm Device: a new technology for rapid determination of antibiotic susceptibilities of bacterial biofilms. J Clin Microbiol 37, 1771-1776.

Characklis, W. G. \& Marshall, K. C. (1990). Biofilms. New York: Wiley. Donlan, R. M., Murga, R., Carpenter, J., Brown, E., Besser, R. \& Fields, B. (2002). Monochloramine disinfection of biofilmassociated Legionella pneumophila in a potable water model system. In Legionella, pp. 406-410. Edited by R. Marre and others. Washington, DC: American Society for Microbiology.

Donlan, R. M., Piede, J. A., Heyes, C. D., Sanii, L., Murga, R., Edmonds, P., El-Sayed, I. \& El-Sayed, M. A. (2004). A model system for growing and quantifying Streptococcus pneumoniae biofilms in situ and in real time. Appl Environ Microbiol 70, 4980-4988.

Feldsine, P., Abeyta, C. \& Andrews, W. H. (2002). AOAC International methods committee guidelines for validation of qualitative and quantitative food microbiological official methods of analysis. J AOAC Int 85, 1187-1200.

Herigstad, B., Hamilton, M. \& Heersink, J. (2001). How to optimize the drop plate method for enumerating bacteria. J Microbiol Methods 44, 121-129.

ISO (1993). Guide to the Expression of Uncertainty in Measurement. Geneva: ISO.

Kharazmi, A., Giwercman, B. \& Hoiby, N. (1999). Robbins Device in biofilm research. Methods Enzymol 310, 207-215.

Mittelman, M. W., Kohring, L. L. \& White, D. C. (1992). Multipurpose laminar-flow adhesion cells for the study of bacterial colonization and biofilm formation. Biofouling 6, 39-51.

Murga, R., Forster, T. S., Brown, E., Pruckler, J. M., Fields, B. S. \& Donlan, R. M. (2001). Role of biofilms in the survival of Legionella pneumophila in a model potable-water system. Microbiology 147, 3121-3126.

Nickel, J. C., Wright, J. B., Ruseska, I., Marrie, T. J., Whitfield, C. \& Costerton, J. W. (1985). Antibiotic resistance of Pseudomonas aeruginosa colonizing a urinary catheter in vitro. Eur $J$ Clin Microbiol 4, 213-218.

NIST/SEMATECH (2003). e-Handbook of Statistical Methods. http:// www.itl.nist.gov/div898/handbook/.

O'Toole, G. A. \& Kolter, R. (1998). Initiation of biofilm formation in Pseudomonas fluorescens WCS365 proceeds via multiple, convergent signaling pathways: a genetic analysis. Mol Microbiol 28, 449-461.

Stoodley, P., Dodds, I., Boyle, J. D. \& Lappin-Scott, H. M. (1999). Influence of hydrodynamics and nutrients on biofilm structure. J Appl Microbiol Symp Suppl 85, 19S-28S.

Thompson, M. (2000). Towards a unified model of errors in analytical measurement. Analyst 125, 2020-2025.

Thompson, M., Ellison, S. \& Wood, R. (2002). Harmonized guidelines for single-laboratory validation of methods of analysis. Pure Appl Chem 74, 835-855.

Youden, W. \& Steiner, E. (1975). Statistical Manual of the Association of Official Analytical Chemists. Gaithersberg, MD: AOAC International.

Zelver, N., Hamilton, M., Pitts, B., Goeres, D., Walker, D., Sturman, P. \& Heersink, J. (1999). Measuring antimicrobial effects on biofilm bacteria: from laboratory to field. Methods Enzymol 310, 608-628. 